\title{
Tumor Occupation in the Spinal Canal and Clinical Symptoms of Cauda Equina Schwannoma: An Analysis of 22 Cases
}

\author{
Kengo Fujii ${ }^{1}$, Masataka Sakane ${ }^{2}$, Tetsuya $\mathrm{Abe}^{1}$, Tsukasa Nakagawa ${ }^{3}$, \\ Shinsuke Sakai ${ }^{4}$, Masaki Tatsumura ${ }^{5}$, Toru Funayama ${ }^{6}$, Masashi Yamazaki ${ }^{1}$ \\ ${ }^{I}$ Department of Orthopaedic Surgery, Faculty of Medicine, University of Tsukuba, Tsukuba, Japan \\ ${ }^{2}$ Department of Rehabilitation, Tsukuba Gakuen Hospital, Tsukuba, Japan \\ ${ }^{3}$ Department of Orthopaedic Surgery, Kensei General Hospital, Sakuragawa, Japan \\ ${ }^{4}$ Department of Orthopaedic Surgery, Tokyo Medical University Ibaraki Medical Center, Inashiki, Japan \\ ${ }^{5}$ Department of Orthopaedic Surgery, Mito Kyodo General Hospital, Mito, Japan \\ ${ }^{6}$ Department of Orthopaedic Surgery, Kenpoku Medical Center Takahagi Kyodo Hospital, Takahagi, Japan
}

Study Design: Retrospective, radiological study.

Purpose: To determine the relationship between clinical symptoms and the extent of tumor occupation of the spinal canal by cauda equina schwannoma.

Overview of Literature: Little is known about the relationship between the size of tumors of the cauda equina and the manifestation of clinical symptoms. We analyzed this relationship by estimating the percentage of tumor occupation (PTO) in the spinal canal in cauda equina schwannomas and by correlating this parameter with the presence and severity of clinical symptoms.

Methods: Twenty-two patients (9 men and 13 women; age, 19-79 years; mean age, 55.3 years) who were radiologically diagnosed with schwannomas of the cauda equina between April 2004 and July 2014 were retrospectively analyzed. PTO was measured in axial and sagittal magnetic resonance imaging slices in which the cross-sectional area of the tumor was the largest. Data regarding clinical symptoms and results of physical examinations were collected from patient medical records. PTO differences between symptompositive and -negative groups were analyzed for each variable.

Results: In the 4 cases in which tumor presence was not related to clinical symptoms, PT0 was 5\%-10\% (mean, 9\%) in axial slices and $23 \%-31 \%$ (mean, 30\%) in sagittal slices. In the 18 cases in which symptoms were associated with the tumor, PT0 was $11 \%-86 \%$ (mean, $50 \%$ ) in axial slices and $43 \%-88 \%$ (mean, $71 \%$ ) in sagittal slices. PTO in axial slices was significantly higher in the presence of Déjèrine symptoms and/or muscle weakness, a positive straight leg raise test, and a positive Kemp sign.

Conclusions: PTO $>20 \%$ in axial slices and $>40 \%$ in sagittal slices can be an indication of symptomatic cauda equina schwannoma.

Keywords: Neurilemmoma; Cauda equina; Surgery

Received Mar 1, 2016; Revised May 26, 2016; Accepted May 27, 2016

Corresponding author: Kengo Fujii

Department of Orthopaedic Surgery, Faculty of Medicine, University of Tsukuba,

1-1-1 Tennodai, Tsukuba 305-8575, Japan

Tel: +81-29-853-3219, Fax: +81-29-853-3162, E-mail: kengox15feb@tsukuba-seikei.jp 


\section{Introduction}

The widespread use of magnetic resonance imaging (MRI) has led to an increase in the discovery of asymptomatic tumors of the cauda equina. Gradual tumor enlargement can cause lumbar canal stenosis, and compression of the cauda equina may lead to neurological damage, thus leading to the development of various symptoms [1]. In general, tumor resection is considered for patients with abnormal neurological findings or progressive symptoms, but precise indications for surgical treatment are not yet clearly established [2]. Because little is known about the relationship between tumor size and clinical symptoms [1], the objective of this study was to determine the relationship between the extent of tumor occupation of the spinal canal and the presence of clinical symptoms in patients with cauda equina schwannoma.

\section{Materials and Methods}

\section{Subjects}

We retrospectively analyzed radiological findings from 22 patients ( 9 men and 13 women; 19 to 79 years old; mean age, 55.3 years) who were radiologically diagnosed with schwannomas in the cauda equina between April 2004 and July 2014. Exclusion criteria were as follows: tumor at or above the conus medullaris, neurofibroma and von Recklinghausen's disease, dumbbell tumor, and other extradural lesions that may affect the symptoms of the patients. Informed consent was obtained from all patients before their participation in this study.

\section{Radiological measurements}

We analyzed axial and sagittal MRI slices (T1-weighted enhanced images or T2-weighted images) in which the cross-sectional area of the tumor was the largest, as shown in Figs. 1 and 2. The percentage of tumor occupation (PTO) in each slice was calculated using Image J software (National Institutes of Health, Bethesda, MD, USA). In sagittal slices, tangents to the cranial and caudal ends of the tumor were drawn perpendicular to the spinal body, as shown in Fig. 2. The cross-sectional area of the spinal canal (area $\mathrm{T}$ ) and the sectional area of the tumor (area C) were measured, and PTO was calculated as area T/area $\mathrm{C} \times 100$.

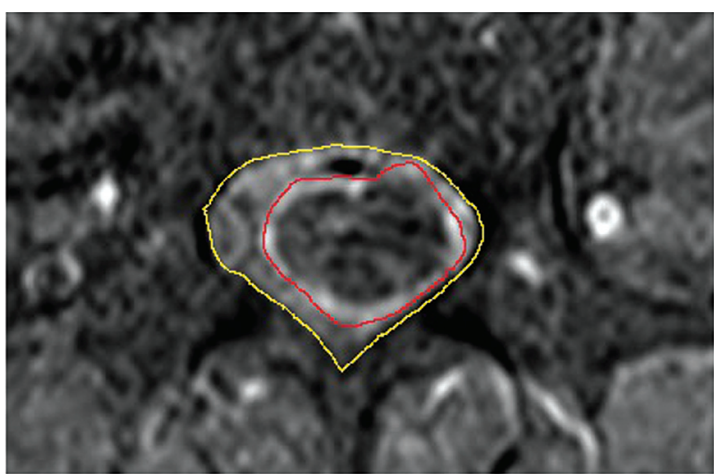

Fig. 1. Measurements and calculations of percentage of tumor occupation (PTO) in an axial magnetic resonance imaging slice. The outer line shows the cross-sectional area of the spinal canal (area C), and the inner line shows the cross-sectional area of the tumor (area T). The pixel count of each area was measured using Image $\mathrm{J}$ software, and PTO was calculated as area T/area $\mathrm{C} \times 100$.

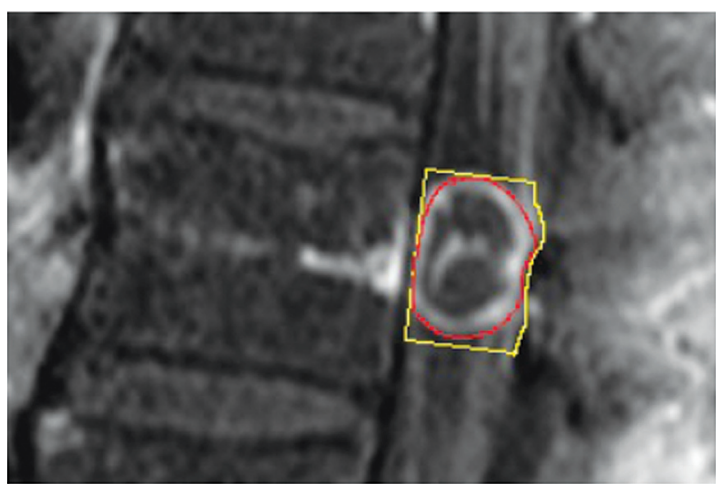

Fig. 2. Measurements and calculations of percentage of tumor occupation (PTO) in a sagittal magnetic resonance imaging slice. The outer line shows the cross-sectional area of the spinal canal (area C), and the inner line shows the cross-sectional area of the tumor (area T). The pixel count of each area was measured using Image $\mathrm{J}$ software, and PTO was calculated as area T/area $\mathrm{C} \times 100$

Whether or not the tumor made contact with the intervertebral discs was also investigated.

\section{Clinical evaluation}

Data regarding the clinical symptoms and results of physical examinations were retrospectively collected from patients' medical records. Tumor-associated symptoms included lower back pain, muscle weakness or sensory disturbance in the lower extremities, bowel or bladder disturbances, night back or leg pain, Déjèrine symptoms, and positive results of the straight leg raise test (SLRT), 
Table 1. The percentage of tumor occupation and clinical symptoms

\begin{tabular}{|c|c|c|c|c|c|c|c|}
\hline \multirow[b]{2}{*}{ Clinical symptoms } & \multicolumn{3}{|c|}{ Positive } & \multicolumn{3}{|c|}{ Negative } & \multirow[b]{2}{*}{$p$-value } \\
\hline & $\begin{array}{l}\text { No. of } \\
\text { cases }\end{array}$ & РTO & SD & $\begin{array}{l}\text { No. of } \\
\text { cases }\end{array}$ & PTO & SD & \\
\hline \multicolumn{8}{|l|}{ Overall } \\
\hline Axial & 18 & 0.48 & 0.17 & 4 & 0.08 & 0.03 & 0.002 \\
\hline Sagittal & 18 & 0.71 & 0.11 & 4 & 0.27 & 0.03 & 0.001 \\
\hline \multicolumn{8}{|l|}{ Symptoms } \\
\hline \multicolumn{8}{|l|}{ LBP } \\
\hline Axial & 9 & 0.34 & 0.16 & 13 & 0.45 & 0.24 & 0.15 \\
\hline Sagittal & 9 & 0.64 & 0.21 & 13 & 0.61 & 0.21 & 0.97 \\
\hline \multicolumn{8}{|l|}{ Muscle weakness } \\
\hline Axial & 6 & 0.46 & 0.07 & 16 & 0.38 & 0.25 & 0.61 \\
\hline Sagittal & 6 & 0.75 & 0.13 & 15 & 0.57 & 0.21 & 0.04 \\
\hline \multicolumn{8}{|l|}{ Sensory disturbance } \\
\hline Axial & 6 & 0.42 & 0.20 & 16 & 0.40 & 0.22 & 0.94 \\
\hline Sagittal & 6 & 0.66 & 0.21 & 15 & 0.61 & 0.21 & 0.38 \\
\hline \multicolumn{8}{|l|}{$\mathrm{BBD}$} \\
\hline Axial & 4 & 0.47 & 0.05 & 18 & 0.39 & 0.23 & 0.61 \\
\hline Sagittal & 4 & 0.72 & 0.15 & 18 & 0.60 & 0.21 & 0.15 \\
\hline \multicolumn{8}{|l|}{ Night pain } \\
\hline Axial & 9 & 0.45 & 0.18 & 13 & 0.37 & 0.24 & 0.40 \\
\hline Sagittal & 9 & 0.70 & 0.19 & 13 & 0.56 & 0.21 & 0.19 \\
\hline \multicolumn{8}{|l|}{ Déjèrine symptoms } \\
\hline Axial & 9 & 0.52 & 0.20 & 13 & 0.32 & 0.19 & 0.04 \\
\hline Sagittal & 9 & 0.69 & 0.14 & 13 & 0.57 & 0.24 & 0.27 \\
\hline \multicolumn{8}{|l|}{ SLRT } \\
\hline Axial & 3 & 0.48 & 0.05 & 19 & 0.39 & 0.23 & 0.47 \\
\hline Sagittal & 3 & 0.80 & 0.04 & 19 & 0.59 & 0.21 & 0.04 \\
\hline \multicolumn{8}{|l|}{ FNST } \\
\hline Axial & 2 & 0.43 & 0.02 & 20 & 0.40 & 0.22 & 0.82 \\
\hline Sagittal & 2 & 0.77 & 0.02 & 20 & 0.60 & 0.21 & 0.21 \\
\hline \multicolumn{8}{|l|}{ Kemp test } \\
\hline Axial & 7 & 0.46 & 0.25 & 15 & 0.38 & 0.20 & 0.75 \\
\hline Sagittal & 7 & 0.71 & 0.18 & 15 & 0.58 & 0.21 & 0.04 \\
\hline
\end{tabular}

PTO, percentage of tumor occupation; SD, standard deviation; LBP, low back pain; BBD, bowel bladder dysfunction; SLRT, straight leg raise test; FNST, femoral nerve stretch test.

femoral nerve stretching test, and the Kemp test. We divided the patients into 2 groups: a symptomatic tumor group and an asymptomatic tumor group. Patients exhibiting symptoms obviously not related to their tumors were classified into the asymptomatic tumor group.

\section{Statistical Analysis}

A Mann-Whitney $U$ test was used to analyze the differences in PTO measurements between the symptom-positive and -negative groups for each clinical variable. $p<0.05$ was considered significant. 


\section{Results}

Tumor location and frequencies of occurrence in our sample were as follows: L2 position in 4 cases, L2-3 in 5 cases, L3 in 3 cases, L3-4 in 4 cases, L4 in 3 cases, L4-5 in 1 case, L5 in 1 case, and S2 in 1 case. Tumors made contact with intervertebral discs in 8 cases.

PTO values (in percentages) are shown in Table 1 and Fig. 3. In the 4 cases comprising the asymptomatic tumor group (in which tumor presence was not related to clinical symptoms), PTO was 5\%-10\% (mean, 9\%) in axial slices and $23 \%-31 \%$ (mean, 30\%) in sagittal slices. In the 18 cases included in the symptomatic tumor group (in which tumor presence was associated with clinical symptoms), PTO was $11 \%-86 \%$ (mean, $50 \%$ ) in axial slices and 43\%-88\% (mean, $71 \%$ ) in sagittal slices.

\section{Relationship between PTO and clinical symptoms}

PTO in axial slices was significantly higher in the presence of Déjèrine symptoms, and PTO in sagittal slices was significantly higher in the presence of muscle weakness, a

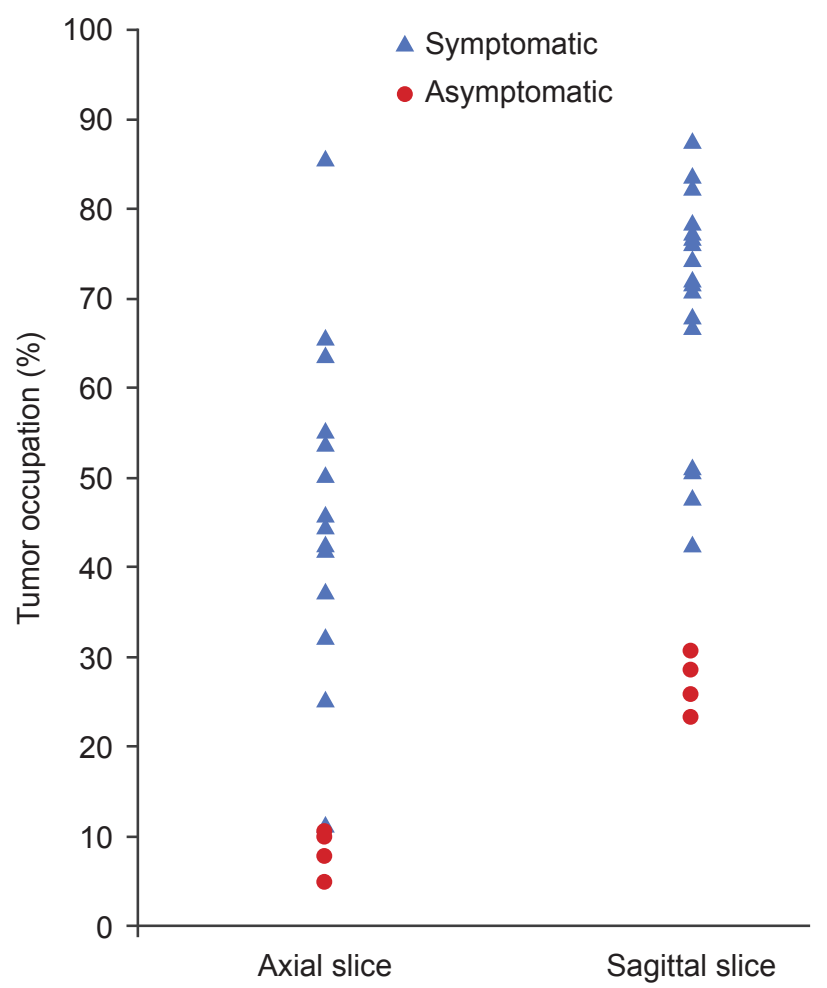

Fig. 3. Percentage of tumor occupation measured in axial and sagittal slices. Red circles correspond to the asymptomatic tumor group, and blue triangles indicate the symptomatic tumor group. positive SLRT, and a positive Kemp sign (Table 1).

\section{Relationship between clinical symptoms and tumor contact with an intervertebral disc}

The presence or absence of tumor contact with intervertebral discs bore no significant relationship with any clinical symptom.

\section{Discussion}

A number of studies have analyzed cross-sectional areas of intracanal-occupying lesions, such as lumbar disc herniation, burst fracture, ligamentum flavum, and ossification of the posterior longitudinal ligament [3-6]. Some authors have reported a correlation of clinical symptoms with both the cross-sectional area of the dural sac and the extent of lumbar canal stenosis [7-13]. However, little is known about the relationship between the clinical symptoms and PTO of cauda equina schwannoma in the lumbar spinal canal [1]. To our knowledge, this is the first study describing a correlation between PTO of cauda equina schwannomas and the associated clinical symptoms.

Our results suggest that PTO values of $>20 \%$ in axial slices and $>40 \%$ in sagittal slices can be indices of symptomatic schwannomas. Furthermore, PTO $>70 \%$ in a sagittal slice can be a rough indicator of the presence of neurological deficit or severe symptoms, such as muscle weakness, a positive SLRT, or a positive Kemp test result. These indices can be used in some clinical situations. First, when PTO is smaller than these indices, we should consider other origins for the patients' symptoms. Second, in cases of asymptomatic cauda equina schwannomas, it can sometimes be very difficult for the patient and doctor to decide the timing of surgical treatment; in these situations, these indices may be useful for clinical planning with regard to determining the follow-up intervals and operative indications. Additional longitudinal studies may reveal the natural progression of tumor enlargement and its association with clinical symptoms.

In general, the cross-sectional area of the spinal canal is narrowest at the level of the intervertebral disc. Therefore, we hypothesized that tumor contact with the intervertebral disc may affect clinical symptoms [14]. Contrary to our hypothesis, the presence or absence of tumor contact with the intervertebral disc had no significant relationship with any clinical symptom. However, since our study 
included a small number of cases, further analyses in a larger number of patients will be necessary to confirm this.

Furthermore, because cauda equina schwannomas may move craniocaudally, we should consider the influence of tumor mobility in the dural sac $[4,15-19]$. Hence, further investigation examining tumor mobility is needed.

Some reports have described dynamic changes in the lumbar dural sac [20-24]. Kanbara et al. [21] reported that bulging of the ligamentum flavum had $50 \%-85 \%$ contribution to spinal canal narrowing. Thus, bulging of intervertebral discs is expected to contribute to spinal canal narrowing under loading conditions by approximately $15 \%-50 \%$. In the present study, we did not examine dynamic factors of the lumbar spinal canal; however, we intend to address this in future studies.

Another concern is the origin of tumors and preoperative or postoperative clinical symptoms. Tumors originating from the ventral nerve root seem to present motordominant symptoms, while those originating from the dorsal nerve root seem to present sensory-dominant symptoms. In this study, we did not examine intraoperative findings (i.e., tumors' origins) or postoperative clinical findings. Further analyses that include these findings are warranted.

Another limitation of this study is that we did not consider the shape or hardness of the tumors or deviations in the spinal canal [4,25-27]. Age, the existence of congenital/degenerative spinal canal stenosis, and vertebral disc pathologies are also important factors that may strongly affect clinical symptoms $[9,13,22,23]$. Tsuji et al. reported that conical herniated discs caused more pain than round herniated discs [25]. Tomita et al. [26] reported that a herniated disc located more laterally caused more pain than a more medially located disc. By contrast, a medially localized herniated disc with a large PTO in its axial slice is likely to indicate bowel and bladder dysfunction [11,27]. Although only schwannoma cases were included in this study, it is likely that tumor density and hardness varied between individual cases. Therefore, three-dimensional tumor shape, hardness, and deviation should be determined in future studies. With regard to age and existence of congenital/degenerative spinal canal stenosis, future studies with a larger number of subjects are warranted to reveal the relationships among age, congenital/degenerative spinal canal stenosis, radiological measurements of tumors, and clinical symptoms.

\section{Conclusions}

PTO $>20 \%$ in axial slices and $>40 \%$ in sagittal slices can be an indication of symptomatic cauda equina schwannomas. Our results suggest that PTO calculated by MRI in axial and sagittal slices can be used to predict the development of clinical symptoms in patients with schwannomas of the cauda equina.

\section{Conflict of Interest}

No potential conflict of interest relevant to this article was reported.

\section{References}

1. Shimada Y, Miyakoshi N, Kasukawa Y, Hongo M, Ando S, Itoi E. Clinical features of cauda equina tumors requiring surgical treatment. Tohoku J Exp Med 2006;209:1-6.

2. Cervoni L, Celli P, Scarpinati M, Cantore G. Neurinomas of the cauda equina clinical analysis of 40 surgical cases. Acta Neurochir (Wien) 1994;127:199202.

3. Chiba K, Kato Y, Tsuzuki N, et al. Computer-assisted measurement of the size of ossification in patients with ossification of the posterior longitudinal ligament in the cervical spine. J Orthop Sci 2005;10:4516.

4. Hur JW, Hur JK, Kwon TH, Park YK, Chung HS, Kim JH. Radiological significance of ligamentum flavum hypertrophy in the occurrence of redundant nerve roots of central lumbar spinal stenosis. J Korean Neurosurg Soc 2012;52:215-20.

5. Ishimoto K, Iguchi T, Kurihara A. Three dimensional analysis of the lumbar disc herniation: relationship between its location, amount of the herniated mass and severity of the symptoms. Rinsho Seikei Geka 1999;34:1197-203.

6. Kaneko K, Omokawa S, Fujitani R. A study of neurological deficits with thoracolumbar burst fractures. Kossetsu 2007;29:499-501.

7. Laurencin CT, Lipson SJ, Senatus P, et al. The stenosis ratio: a new tool for the diagnosis of degenerative spinal stenosis. Int J Surg Investig 1999;1:127-31.

8. Chung SW, Kang MS, Shin YH, Baek OK, Lee SH. Postoperative expansion of dural sac cross-sectional 
area after unilateral laminotomy for bilateral decompression: correlation with clinical symptoms. Korean J Spine 2014;11:227-31.

9. Hamanishi C, Matukura N, Fujita M, Tomihara M, Tanaka S. Cross-sectional area of the stenotic lumbar dural tube measured from the transverse views of magnetic resonance imaging. J Spinal Disord 1994;7: 388-93.

10. Genevay S, Atlas SJ. Lumbar spinal stenosis. Best Pract Res Clin Rheumatol 2010;24:253-65.

11. Inui Y, Doita M, Ouchi K, Tsukuda M, Fujita N, Kurosaka $\mathrm{M}$. Clinical and radiologic features of lumbar spinal stenosis and disc herniation with neuropathic bladder. Spine (Phila Pa 1976) 2004;29:869-73.

12. Steurer J, Roner S, Gnannt R, Hodler J. Quantitative radiologic criteria for the diagnosis of lumbar spinal stenosis: a systematic literature review. BMC Musculoskelet Disord 2011;12:175.

13. Ogikubo O, Forsberg L, Hansson T. The relationship between the cross-sectional area of the cauda equina and the preoperative symptoms in central lumbar spinal stenosis. Spine (Phila Pa 1976) 2007;32:1423-8.

14. Liu SY, Lin YM, Wei TS, Lin SJ, Liu CC, Chou CW. Exacerbation of symptoms of lumbar disc herniation complicated by a schwannoma: a case report. Kaohsiung J Med Sci 2007;23:480-5.

15. Satoh T, Kageyama T, Kamata I, Date I. Mobile neurinoma of the cauda equina; a case report. No Shinkei Geka 1991;19:891-6.

16. Hollin SA, Drapkin AJ, Wancier J, Huang YP. Mobile schwannoma of the cauda equina: case report. J Neurosurg 1978;48:135-7.

17. Isu T, Iwasaki Y, Akino M, Nagashima M, Abe $H$. Mobile schwannoma of the cauda equina diagnosed by magnetic resonance imaging. Neurosurgery 1989; 25:968-71.

18. Sasaki M, Aoki M, Yoshimine T. Mobile schwannoma of the cauda equina incarcerated following caudal migration after trauma: case report. Neurol Med Chir (Tokyo) 2011;51:710-2.

19. Marin-Sanabria EA, Sih IM, Tan KK, Tan JS. Mobile cauda equina schwannomas. Singapore Med J 2007; 48:e53-6.

20. Hansson T, Suzuki N, Hebelka H, Gaulitz A. The narrowing of the lumbar spinal canal during loaded MRI: the effects of the disc and ligamentum flavum. Eur Spine J 2009;18:679-86.

21. Kanbara S, Yukawa Y, Ito K, Machino M, Kato F. Dynamic changes in the dural sac of patients with lumbar canal stenosis evaluated by multidetector-row computed tomography after myelography. Eur Spine J 2014;23:74-9.

22. Kanno H, Ozawa H, Koizumi Y, et al. Dynamic change of dural sac cross-sectional area in axial loaded magnetic resonance imaging correlates with the severity of clinical symptoms in patients with lumbar spinal canal stenosis. Spine (Phila Pa 1976) 2012;37:207-13.

23. Kanno H, Endo T, Ozawa H, et al. Axial loading during magnetic resonance imaging in patients with lumbar spinal canal stenosis: does it reproduce the positional change of the dural sac detected by upright myelography? Spine (Phila Pa 1976) 2012;37:E98592.

24. Ito K, Yukawa Y, Machino M, et al. Dynamic changes in the spinal cord cross-sectional area in patients with myelopathy due to cervical ossification of posterior longitudinal ligament. Spine J 2015;15:461-6.

25. Tsuji H, Toyotomi S. Theoretical perspective of intraneural stress patterns in a compressed nerve root and basic concept of nerve root decompression with special reference to herniated nucleus pulposus in aged patients. Rinsho Seikei Geka 1987;22:411-6.

26. Tomita S, Furufu T, Sakamoto M. The investigation MRI findings in lumbar intervertebral disc herniation: enhanced pattern of conservative and surgical treatment. Orthop Surg (Seikeigeka) 1997;48:1323-7.

27. Tsai CH, Chou EC, Chou LW, et al. The evaluation of bladder symptoms in patients with lumbar compression disorders who have undergone decompressive surgery. Spine (Phila Pa 1976) 2010;35:E849-54. 